\title{
Téoros
}

Revue de recherche en tourisme

\section{Wright et Le Corbusier, figures emblématiques de la mise en tourisme des architectures du $\mathrm{XX}^{\mathrm{e}}$ siècle}

\section{Gabriel Rioux}

Volume 21, numéro 2, été 2002

Patrimoines du $\mathrm{XX}^{\mathrm{e}}$ siècle

URI : https://id.erudit.org/iderudit/1072405ar

DOI : https://doi.org/10.7202/1072405ar

Aller au sommaire du numéro

Éditeur(s)

Université du Québec à Montréal

ISSN

0712-8657 (imprimé)

1923-2705 (numérique)

Découvrir la revue

Citer cet article

Rioux, G. (2002). Wright et Le Corbusier, figures emblématiques de la mise en tourisme des architectures du XX ${ }^{\mathrm{e}}$ siècle. Téoros, 21(2), 32-39.

https://doi.org/10.7202/1072405ar d'utilisation que vous pouvez consulter en ligne. 


\section{Wright et Le Corbusier, figures emblématiques de la mise en tourisme des architectures du $\mathrm{XX}^{\mathrm{e}}$ siècle}

\section{Gabriel Rioux}

$\mathbf{T}$ ourisme et architecture : ces mots aussitôt prononcés, le contact s'opère. Celui du visiteur, parcourant un lieu, une œuvre, qui entre en communion avec l'identité, parfois les idéaux d'une culture. L'effervescence du mouvement moderne, attisé par des architectes éblouis devant les avancées technologiques de la société industrielle, s'est traduite par la création de nouveaux cadres de vie. Parmi les nombreux acteurs d'avant-garde, deux noms s'imposent : Frank Lloyd Wright (18671959) et Charles-Édouard Jeanneret, dit Le Corbusier (1887-1965). Pour chacun, c'est l'occasion de redéfinir les standards de vie dans la société, d'exprimer la forme issue de l'avènement d'un temps nouveau, une profonde refonte des valeurs, comme un écho à l'appel de l'homme supérieur de Nietzsche. Dans l'ensemble de leur œuvre respective, deux édifices évoquent l'essence de la recherche des maittres. D'abord la villa Savoye (1928-1931) de Le Corbusier, suivie chronologiquement de la maison sur la Cascade (1936-1939) de Frank Lloyd Wright. Les architectes successeurs, les héritiers de ces œuvres, n'ont pu ignorer la matérialisation de leurs convictions. Depuis leur création, le mouvement moderne s'est métamorphosé. Dans l'« impermanence » des choses, les deux édifices ont changé de vocation et sont, depuis peu, accessibles au grand public. À la lumière du discours des créateurs et des historiens, nous pouvons constater que leur mise en valeur touristique marque également une étape cruciale et révélatrice quant au traitement que l'on réserve à ces icônes de l'architecture. Au-delà des acquis, c'est la cohésion qu'ils suscitent qui sera au centre des préoccupations de cette découverte commentée.

\section{Fallingwater (1936-1939)}

L'année 1936 marque un tournant décisif dans la carrière de Wright : la maison sur la Cascade (Fallingwater ou maison Kaufmann) prend forme et devient rapidement une œuvre célèbre. Héritier d'élection du célèbre architecte Louis Sullivan, Wright expérimente dès la fin du $\mathrm{XIX}^{\mathrm{e}}$ siècle la construction de résidences particulières. En réaction aux méfaits de la ville chaotique du moment, il propose une vision complémentaire du romantisme et de l'évolution industrielle. C'est l'architecture intégrée à son environnement, ou organique, qui est d'abord l'inspiration des « maisons de la Prairie » de 1900 à 1911. Ses voyages au Japon alimentent sa recherche d'une architecture qui accorde l'homme avec la nature et lui offrent l'occasion d'expérimenter des techniques constructives inhabituelles, comme la dalle avec cantilever ${ }^{1}$ mise à l'essai à l'Hôtel Impérial de Tokyo (1915). À l'héritage du mouvement pittoresque à l'anglaise se superpose donc un bagage oriental pour créer un langage architectural original.

En 1930, Wright est ruiné, sa vie familiale est brisée ; il est perçu comme un créateur épuisé. L'architecte entreprend alors un redressement décisif de sa carrière en publiant des ouvrages qui interpellent, entre autres, Edgar Kaufmann, richissime marchand de Pittsburgh. La commande de Fallingwater est due au mécénat des Kaufmann ; conçue dans un climat de confiance et dotée de moyens, elle donne naissance à une œuvre charnière dans la carrière de Wright. Surplombant une cascade de la rivière Bear Run, avec ses larges terrasses posées en encorbellement autour d'une cheminée centrale, Fallingwater charme aussitôt l'Amérique qui y voit l'expression du génie national' ${ }^{2}$. En fait, Wright a créé une œuvre qui fait avant tout appel à la sensibilité et à la force de l'observateur ${ }^{3}$. Ainsi, la maison, avec son fenêtrage horizontal inséré entre les dalles et les terrasses s'ouvre largement sur la nature environnante, abolissant en quelque sorte l'habituelle distinction entre intérieur et extérieur. Il s'agit de l'ultime déclaration romantique de Wright qui, aux yeux de ses contemporains témoigne là du renouvellement de sa capacité créative ; avec Fallingwater Wright entame la période la plus productive de sa carrière. Le célèbre Johnson Wax Administration Building (1936-1939)4, érigé à la même époque devient en effet une figure tout aussi emblématique de l'Amérique.

Fallingwater naît d'années de recherches sur la plasticité de la forme, que permet l'utilisation du béton armé, mais aussi sur l'usage de matériaux naturels, plus légitimes aux yeux du créateur parce qu'issus du « lieu » de l'architecture. La conception de Fallingwater émerge aussi des réflexions de l'architecte sur Usonia, société utopique dont il fait la promotion via des maisons modernes usinées, aux toits plats. Les maisons usoniennes prêtent vie à la Broadacre City, modèle prophétique d'une cité-nation démocratique où tous auraient un accès à la propriété parce que la ville s'étalerait sur l'ensemble du territoire quadrillé étasunien.

La maison sur la Cascade sert de résidence secondaire aux Kaufmann jusqu'à l'a- 
près-guerre. L'héritier, Edgar Kaufmann junior, nourrit très tôt l'espoir d'en faire un lieu public et cède, en 1963, la maison à la Western Pennsylvania Conservancy (WPC). Membre de l'organisme sans but lucratif dès les débuts, Edgar Kaufmann junior a aussi participé au succès de l'étape de transition en formulant des orientations assurant le respect des vœux de ses parents et de ceux du créateur. Dès 1964, le site est accessible aux visiteurs. La WPC, appuyée à l'occasion par des spécialistes, notamment de la Fondation Frank Lloyd Wright, mène le projet depuis ce jour.

\section{La maison sur la Cascade, au centre du tissu de la Disappearing City}

À deux heures trente de route de Pittsburgh, dans les vallées verdoyantes de la Pennsylvanie, le site de la maison sur la Cascade est depuis ses débuts un lieu couru du patrimoine moderne américain. La forêt mature accorde le visiteur au contact attendu avec le symbole vivant de l'architecture organique. Les installations d'accueil surprennent. D'abord par la qualité de leur intégration au milieu, avec les pavillons sis au pied des grands pins. Ensuite par la variété des services qui sont offerts : salle d'exposition, librairie et boutique de souvenirs, restauration, garderie ainsi que les commodités usuelles. Enfin, au comptoir d'accueil, les préposés étalent 1'ensemble des visites offertes : le tour complet pour les plus hardis, les activités spéciales pour les enfants, la randonnée nature, les tours « découverte » d'une heure et le tour des membres couronné par un repas sur la terrasse privée.

Le site, fier de ses 2000 hectares, est pourvu de sentiers sinueux qui permettent au promeneur de déambuler sans perturber l'environnement fragile des sous-bois. Porté par le chant continu des oiseaux, le visiteur, soudain plus attentif, est bercé par l'atmosphère des lieux. Les sentiers dévalent un escarpement qui mène sans effort à la maison tant attendue. C'est d'abord le courant de l'eau qui attire l'attention, puis la maison apparaît dans toute sa splendeur, avec ses traits caractéristiques. Le guide accompagne le groupe bigarré qui s'émer-
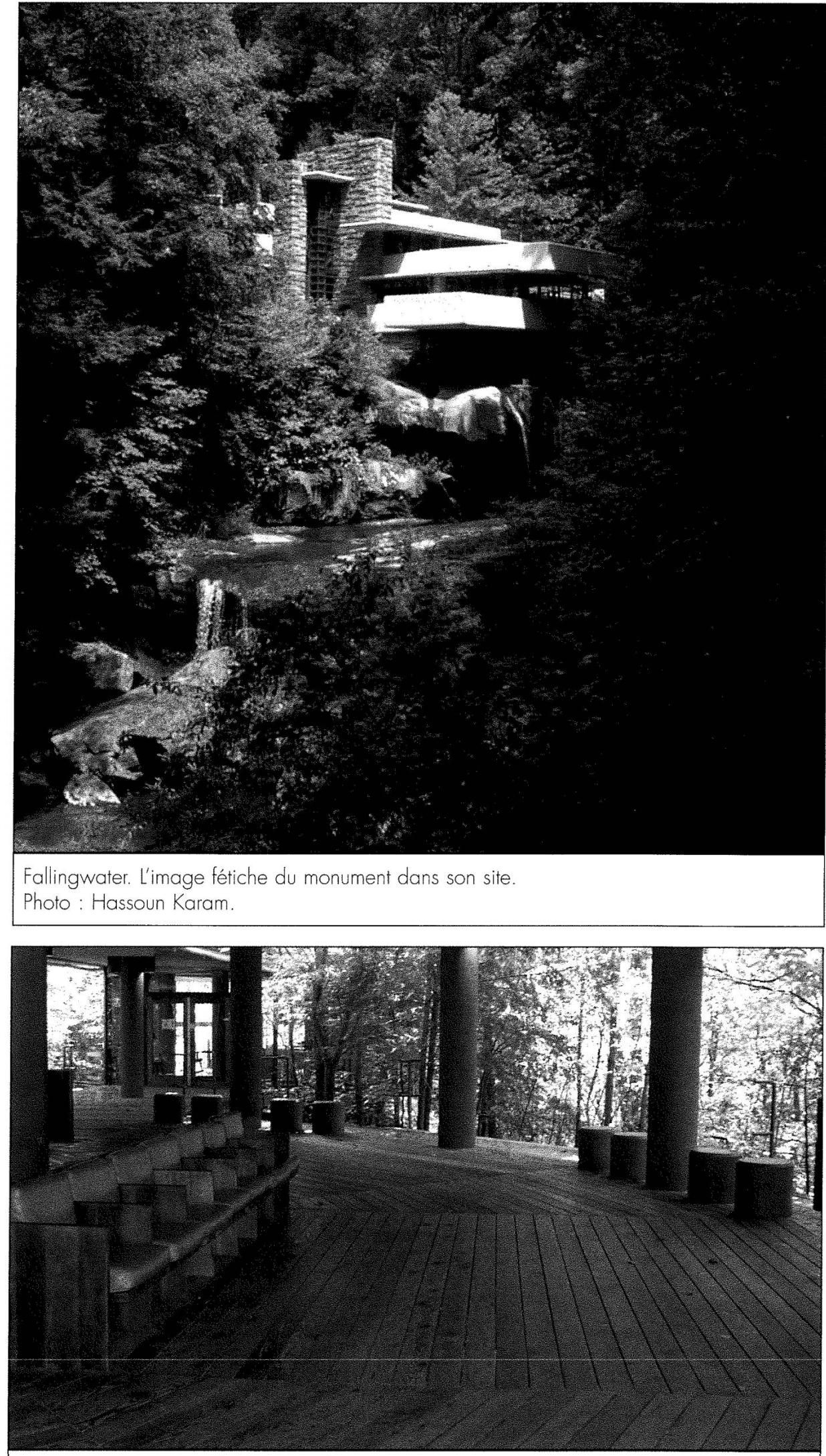

Fallingwater. Le pavillon d'accueil.

Photo: Hassoun Karam. 


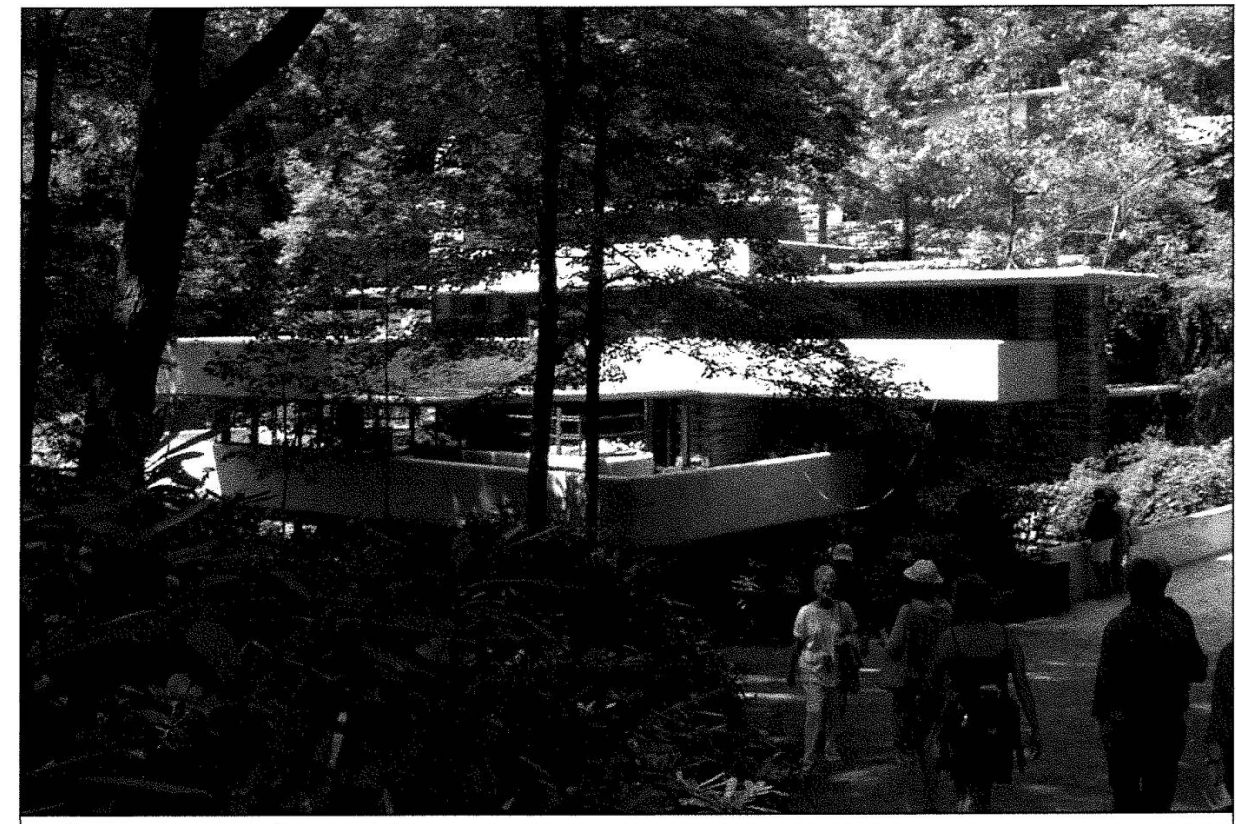

Fallingwater. Vue d'ensemble.

Photo: Hassoun Karam.

veille tout en découvrant les détails de l'histoire du lieu et qui découvre, une fois à l'intérieur, une ambiance tantôt caverneuse dans les corridors, puis chaleureuse dans les appartements généralement exigus. La maison est meublée et toutes ses pièces sont parées des œuvres et des accessoires dont les Kaufmann se servaient.
Au rythme des explications du guide, qui orchestre avec précision la visite, le visiteur est inondé d'information alors que ses yeux tentent de cerner tous les détails. À l'intérieur, les règles sont strictes, le flux incessant des groupes oblige les observateurs à suivre le guide qui s'efforce de répondre aux interrogations sans retarder les groupes qui succèdent. Rien de surprenant quand on sait que 145000 visiteurs accèdent chaque année à la maison. De ce nombre, à peine $20 \%$ sont étrangers. Grâce aux audio-guides et aux documents adaptés, même ceux qui connaissent peu l'anglais y trouvent leur compte. Le parcours se termine à la maison des invités qui jouxte le bâtiment principal. La visite prend fin dans une salle de projection où la WPC procède à une séance destinée à recruter des membres supporters. Ce qui étonne le plus ce n'est pas d'avoir droit à une séance bien légitime de recrutement, c'est plutôt d'apprendre que cette salle de projection, aménagée fidèlement dans le style Wright, était à l'origine un garage ! Ici le visiteur ne se formalise pas d'un tel arrangement, alors qu'un tel geste serait difficilement admissible de l'autre côté de 1'Atlantique où il serait perçu comme portant atteinte à l'intégrité de l'œuvre architecturale. Avec simplicité, Edgar Kaufmann junior donne l'explication d'une telle mise en valeur «Could Fallingwater stay vital? - not a relic but a witness for the future » (1986: 65). Là où les voitures rutilantes des Kaufmann s'immobilisaient il n'y a pas si longtemps, les groupes défilent aujourd'hui à vive allure. La WPC a su tirer profit de la renommée de l'édifice en sensibilisant les
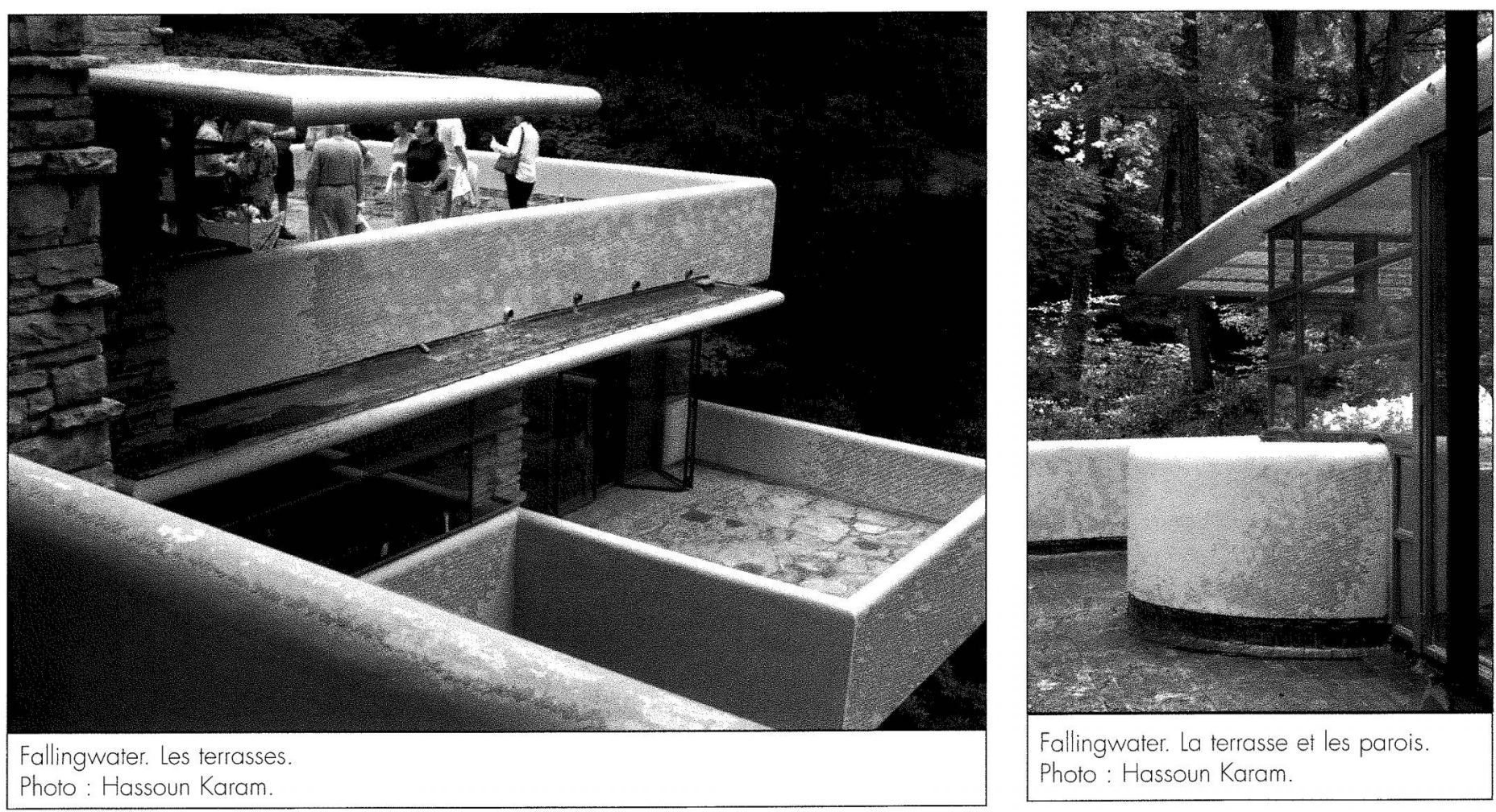
visiteurs à leur cause première : l'écologie et la conservation des espaces naturels; et c'est en remplaçant les voitures des Kaufmann qu'ils donnent le ton.

Le site est périodiquement le lieu d'activités culturelles diverses : concerts, performances professionnelles et rencontres d'associations. De plus, moyennant l'allègement de son compte bancaire, il est possible, si son horaire le permet, de louer la maison pour une utilisation privée. C'est malgré tout le souci de rendre le site accessible au grand public qui prime et les ac- tivités spéciales s'insèrent à intervalle dans les creux de l'année. Interrogés sur la viabilité de l'opération, les responsables de la WPC répondent sans équivoque que l'attraction s'autofinance. Ils font d'ailleurs bien peu de cas du classement patrimonial dont l'édifice bénéficie et ils n'attendent pas les subsides de l'État pour vivre; le site a vraisemblablement atteint une maturité dans sa mise en valeur touristique. Interrogés sur les orientations des dix prochaines années, les porte-parole de la WPC ont semblé soudain plus songeurs, confessant enfin que l'amélioration du paysage-
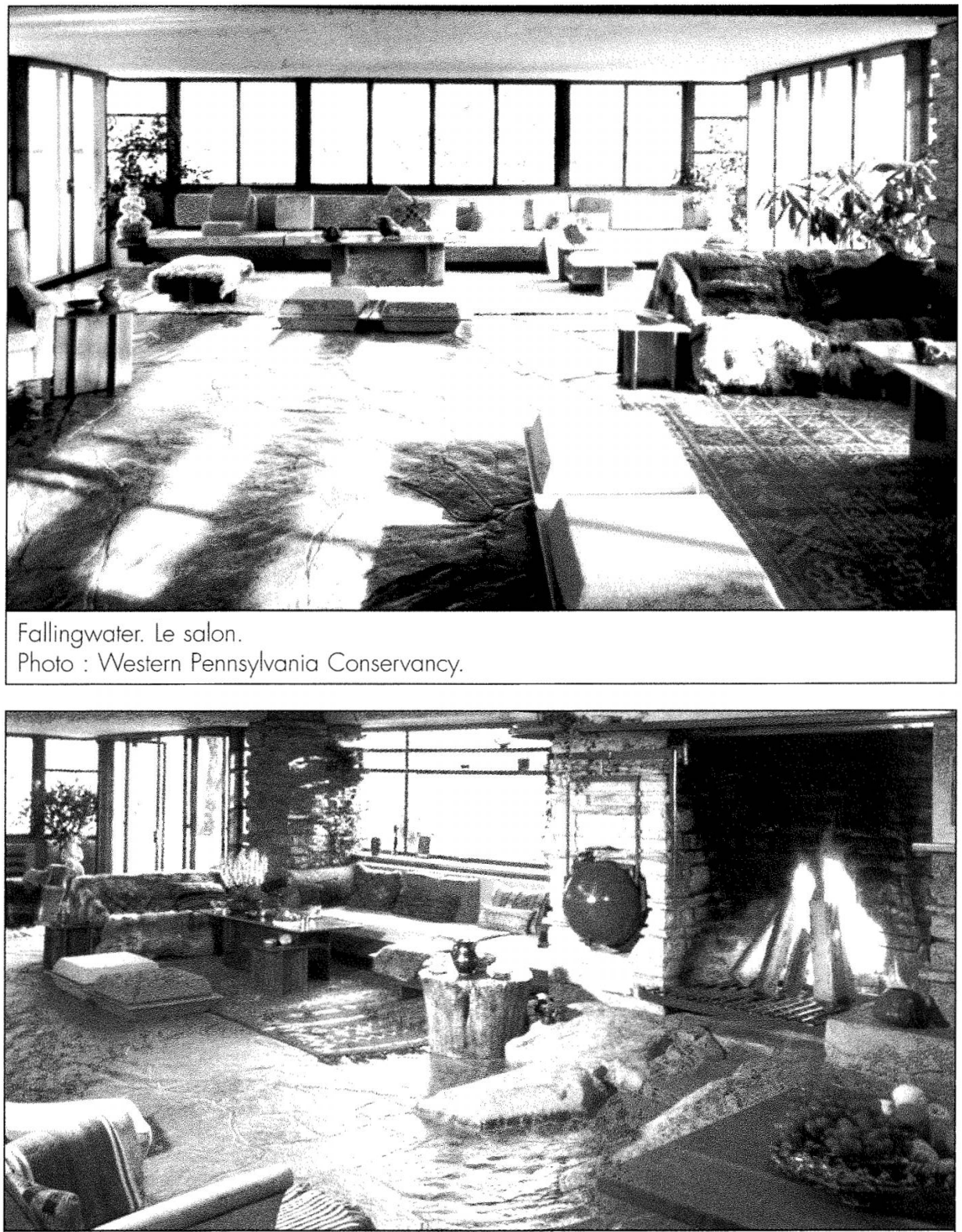

Fallingwater. Le salon et l'âtre.

Photo: Western Pennsylvania Conservancy.

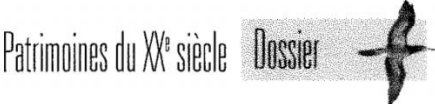

ment, du stationnement et des systèmes d'irrigation faisaient partie des priorités. Les installations existantes ont pourtant fière allure. Décidément, la WPC semble en accord avec l'avis de Wright - qui commentait sa création - « Fallingwater est une grande bénédiction parmi celles que l'on peut recevoir ici sur terre ${ }^{5} \gg$.

\section{La villa Savoye (1928-1931)}

Dernière de la lignée des villas blanches parisiennes, dites « villas puristes », la villa Savoye de Le Corbusier incarne la forme la plus achevée du langage architectural développé dans Vers une architecture $(1923)^{6}$. Elle est, dans la quête du créateur, l'œuvre qui témoigne de la maturité, de l'assurance du jeune architecte radical qui s'inscrit, dès lors, comme le maître de la forme? Trente kilomètres de voiture séparent la villa de Poissy du centre parisien ; l'intégration de l'automobile, dans l'œuvre de Le Corbusier, atteint son point d'orgue à la villa Savoye. La structure «s'élève » et cède la place à l'automobile qui se faufile sous les fragiles pilotis. Ainsi, c'est le court rayon de braquage de la Citroën française qui dicte la dimension de la villa, munie d'un garage où s'engouffrent trois de ces délicats véhicules. Ici l'harmonie naît du contraste de la géométrie de la composition insérée dans l'environnement maitrisé. Mais c'est du jardin, projeté sur le toit, que l'homme entre en contact avec la nature, à travers des tableaux ouverts qui encadrent des instants de paysage. Si de l'extérieur, la villa Savoye adopte une figure hiératique, digne du temple classique, à l'intérieur, les aménagements fonctionnels témoignent de la volonté de répondre de façon rationnelle aux besoins des occupants.

Intellectuel, artiste aux talents multiples, Le Corbusier est très tôt inspiré par les progrès techniques de son époque ; sa conception incarne avec brio les principes et procédés les plus novateurs. Polémiste, il prône l'utilisation de matériaux nouveaux et rejette les styles historiques, référents omniprésents chez ses prédécesseurs et contemporains. Préconisant la standardisation des méthodes de production dans l'habitat moderne, il utilise une rhétorique rationnelle pour enflammer son auditoire. C'est ainsi qu'il 
affirme que les formes primaires de la villa Savoye s'accordent aux lois rigoureuses de la nature. Mais la commande des Savoye rejoint un architecte qui se remet en question. En effet, dès 1928, voyant qu'il ne peut répondre aux besoins de logement social avec une typologie de villas issue de son prototype de la maison Citrohan, dont la villa Savoye est une variante, l'architecte s'engage plutôt dans la poursuite du projet de Cité radieuse, qui donnera naissance à plusieurs imposantes unités d'habitations. La villa Savoye acquiert alors le statut de manifeste, objet qui incarne un temps de l'évolution de la pensée de l'auteur de la « machine à habiter».

\section{Dans I'adversité la maison survit}

En mars 1959, la revue Time Magazine présente au grand public l'état de la villa Savoye : «La machine à habiter est devenue une machine pour cultiver. Elle est transformée en grange à foin ${ }^{8} »$. Après une courte occupation des Savoye à partir de 1932, la villa présente quelques avaries, réparées par les concepteurs jusqu'en 1937. Puis, pour les besoins de la guerre, la villa est réquisitionnée par les Allemands, suivis des alliés, qui en font un poste de garde. Au lendemain du conflit, la dégradation de l'édifice est avancée, les activités agricoles et des occupations sporadiques ont laissé de sérieuses balafres à l'armature dépouillée. La commune de Poissy s'enquiert donc de la situation et suggère la démolition du bâtiment afin d'utiliser les terrains pour l'établissement d'un lycée. Des procédures d'expropriation sont entamées en 1956, mais une mobilisation importante est orchestrée par Le Corbusier et des appuis décisifs de l'étranger forcent le ministre de la Culture, André Malraux, à intervenir. L'édifice est classé aux Bâtiments civils en 1964, puis, peu de temps après la mort de Le Corbusier, aux Monuments historiques? Suivra un éprouvant chantier de restauration qui durera plus de trente-cinq ans. Jusqu'au milieu des années 1980, l'état de conservation de la villa apparaît médiocre aux visiteurs ${ }^{10}$ et ce n'est que depuis 1997 que le public peut la redécouvrir dans son «état d'origine ${ }^{11}$ ».

\section{À Poissy, \\ une visite de la villa Savoye}

Accueilli par la familière loge du gardien, passant par l'entrée grillagée, le visiteur de la villa Savoye pressent dès lors qu'il s'agit d'une visite pour public averti. Un simple écriteau signale au marcheur que la villa n'est pas très loin. Après quelques pas, la blancheur immaculée de la villa transparaît au travers du boisé puis, à l'approche, chaque détail se dévoile. Posée au milieu de l'espace découvert, contrastant avec l'herbe verte, la « Villa aux heures claires ${ }^{12}$ » stupéfait le spectateur qui préfère d'abord l'apprivoiser à distance. Ce n'est qu'une fois à l'intérieur qu'il se rassure. Un petit accueil, aménagé sur le comptoir intégré de l'entrée, se révèle discrètement. Quelques ouvrages ornent la table du guichet d'entrée. Le droit d'entrée payé, la maison est livrée au visiteur. La première pièce visitée propose quelques sobres panneaux à la lectu-
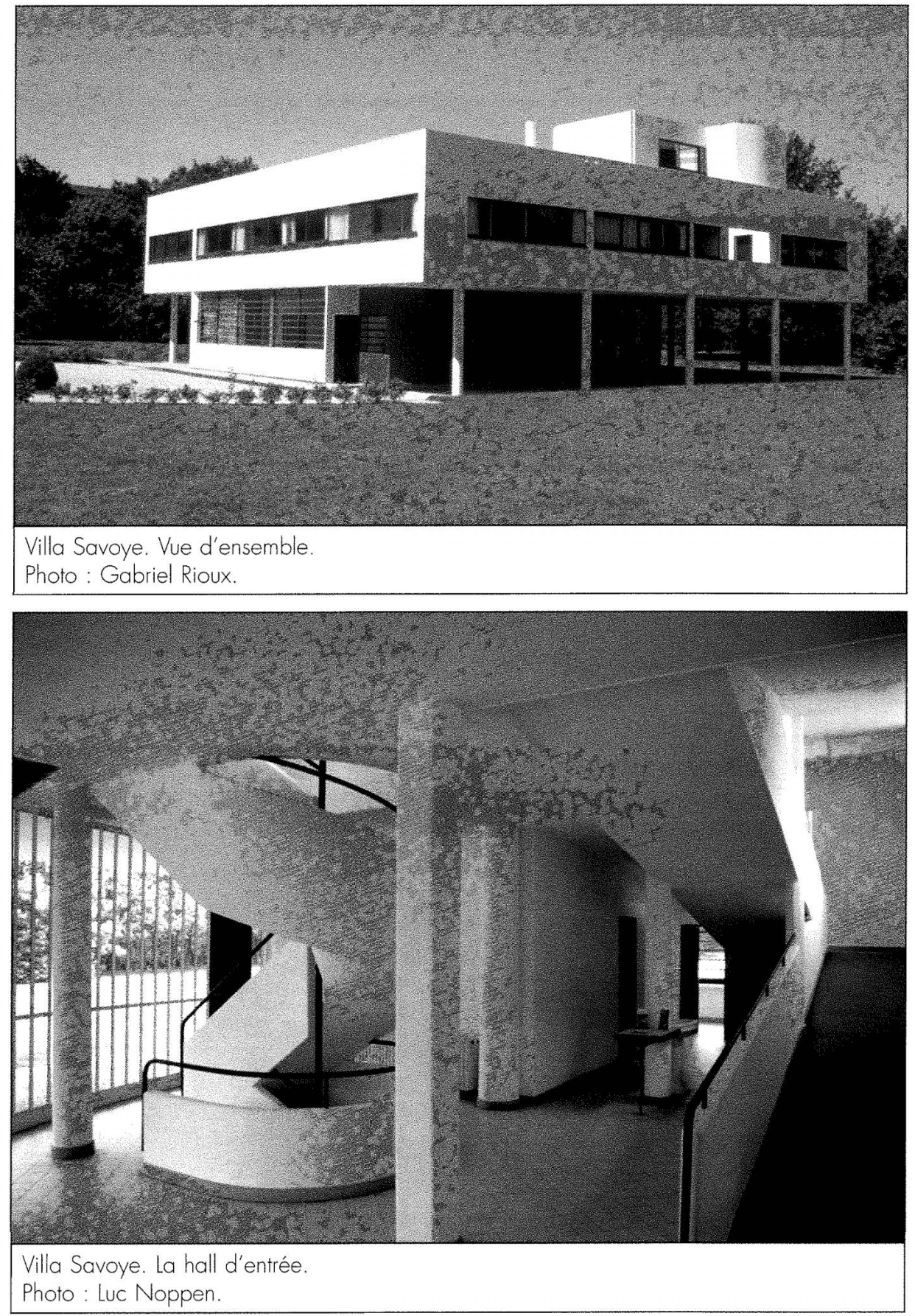
re. Partout ailleurs, c'est à la fois le musée et l'œuvre que découvre le visiteur. Chaque pas lui fait découvrir la richesse sémantique des lieux : «[...] il s'agit d'une véritable promenade architecturale, offrant des

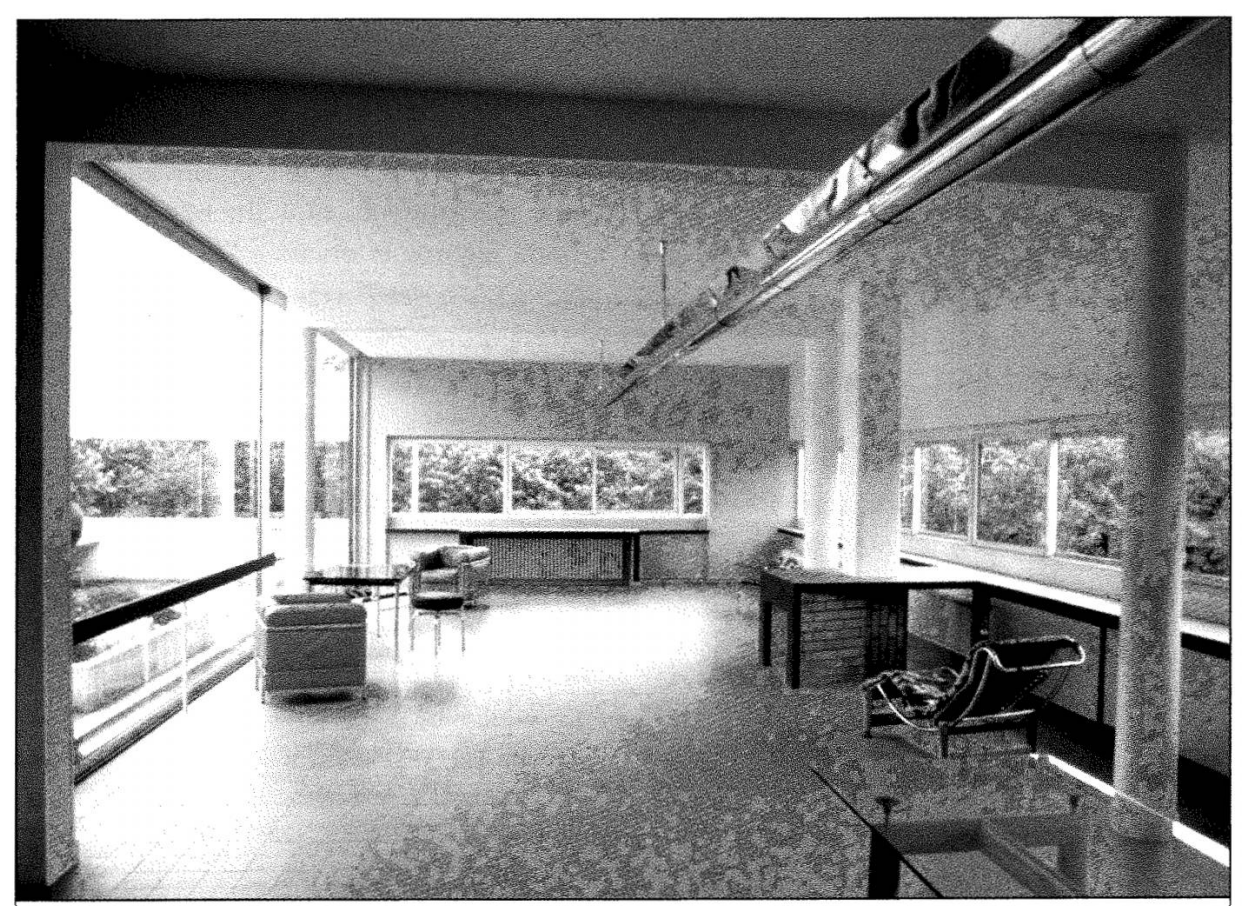

Villa Savoye. Le grand salon qui s'ouvre sur la terrasse à l'étage. Photo: Luc Noppen.

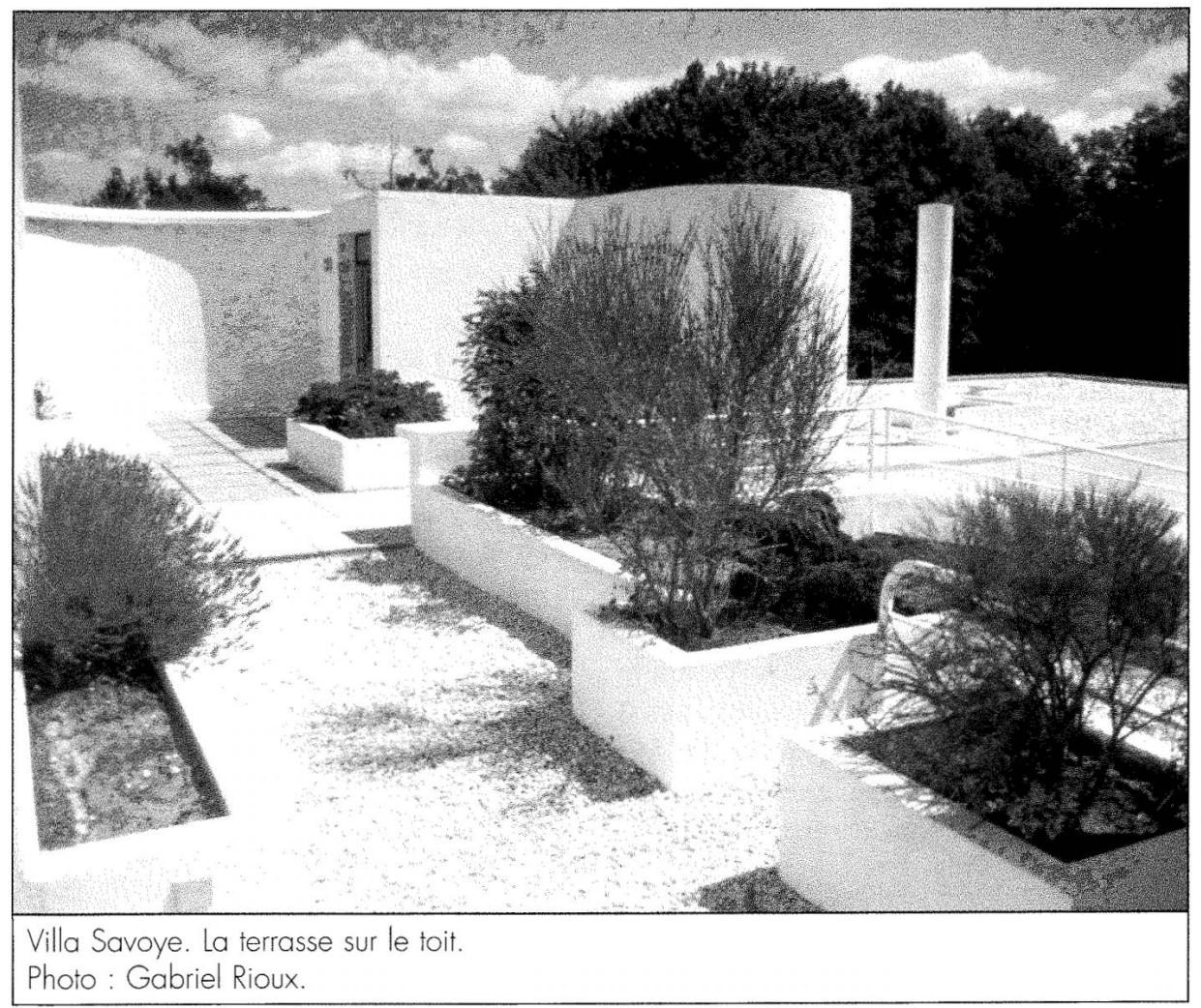

aspects constamment variés, inattendus, parfois étonnants » (Le Corbusier, 1948).

La Villa aux heures claires réjouit le photographe qui peut s'adonner à une séance de

.

s. président de la Fondation Le Corbusier, Claude Prélorenzo, «Toute intervention sur un édifice de Le Corbusier s'opère sous des regards vigilants, sur une scène fortement éclairée » (Verret, 1990 : 12). Mais les difficultés et le coût de l'entreprise de restauration semblent avoir eu raison de la volonté de l'État à entreprendre une seconde étape : la mise en valeur touristique. Le projet s'est avéré un gouffre financier et les droits d'entrée perçus ne permettent pas d'éponger un déficit d'opération récurrent, assumé par l'État depuis qu'il a pris charge du monument.

La villa attire un public averti. Soixante pour cent des visiteurs proviennent de l'étranger et plus de la moitié sont issus des milieux académiques. Mais en regard de la notoriété internationale du lieu, notamment du fait de sa position exemplaire dans l'histoire de l'architecture moderne en Occident, il faut reconnaitre que le site, avec ses 20000 visiteurs annuels, n'est pas très achalandé. Légèrement excentrée, les tren- 
te kilomètres qui séparent la villa du noyau parisien ne l'isolent pas pour autant de l'effervescence touristique de la métropole. Accessible par les transports en commun, elle est à moins d'une heure de l'île de la Cité. Quel contraste avec Fallingwater, située à 100 kilomètres de Pittsburgh, uniquement accessible en voiture ! La villa Savoye est-elle noyée dans le volume d'attractions dont regorge la Ville lumière ? Même les publics des institutions locales, comme celui du Lycée Le Corbusier, qui a grugé une bonne partie du terrain d'origine, sont peu enclins à fréquenter les lieux. $\mathrm{Au}$ ministère de la Culture et de la Communication, une perche est tendue : la gestion locale est envisagée afin d'inciter le milieu à animer la villa. La direction de la Fondation Le Corbusier souhaite que sa mise en valeur touristique soit confiée à un nouveau partenaire et une réflexion a déjà été entamée à ce sujet lors des Rencontres de la Fondation Le Corbusier au début des années 1990. Restaurée depuis, la villa Savoye peut aujourd'hui apparaître plus intéressante à des partenaires désireux d'assurer une mise en tourisme plus dynamique du site.

À lui seul, l'aménagement paysager du site ne contribue guère à la visite ; rien n'y incite le visiteur à prendre quelques instants de repos. L'installation d'un mobilier et l'aménagement d'un parcours permettraient aux visiteurs de faire une pause, de contempler la maison plus longuement. Dans la maison, le garage, si caractéristique avec sa longue porte sur rail, pourrait être utilisé autrement que comme vestiaire. En effet, en l'absence de visites guidées - disponibles seulement sur rendez-vous - il serait possible d'en faire une pièce de projection où le visiteur pourrait découvrir, à travers ses nombreux témoignages enregistrés, la verve de Le Corbusier. On pourrait aussi y utiliser des modèles numériques 3D, matériel apprécié par les clientèles plus jeunes, pour interpréter les qualités constructives et spatiales de la villa. Ces propositions, malgré leur forme primaire, n'ont d'autre but que de remédier à une présentation par trop statique. Avant d'envisager une mise en réseau avec les autres attractions de la commune de Poissy et de la région parisienne, il faut d'abord songer à un programme d'animation plus dynamique qui rejoigne la sensibilité des visiteurs de notre époque. Il est important de comprendre que si pour la plupart des visiteurs d'aujourd'hui Le Corbusier est un héros encore vivant, dans quelques années la simple évocation du nom de l'architecte ne suffira plus pour attirer une clientèle, même réduite.

\section{Conclusion}

D’emblée, nous avons pu constater que les deux monuments - véritables manifestes du renouveau de l'art d'habiter au $\mathrm{XX}^{\mathrm{e}}$ siècle - font l'objet d'une mise en tourisme fort différente. Observateurs et interprètes de la crise sociale de leur époque, Wright et Le Corbusier ont formulé des solutions souvent diamétralement opposées. De ce fait, comme l'exprime avec clarté Robert Fishman (1977 : 127), «Il semble que Wright et Le Corbusier soient prédestinés à être comparés». Les prototypes de maison idéale que sont les villas Savoye et Fallingwater, nous révèlent encore aujourd'hui l'énergie créatrice de leurs auteurs.

À Fallingwater, loin de souscrire à une mise en valeur touristique tous azimuts, c'est avec doigté que les administrateurs ont pu concilier le respect des qualités de l'édifice et des valeurs qu'il permet d'interpréter avec le changement de statut du lieu. Dans l'espoir de perpétuer le mouvement qui est à l'origine de la création de Fallingwater, Edgar Kaufmann junior a insisté sur une distinction fondamentale : « [...] preservation is stopping life to serve a future contingence; conservation is keeping life going » (Kaufmann, 1989:66). La maison de l'élite s'est ainsi métamorphosée en bastion de la culture populaire étasunienne. Elle participe clairement à la quête identitaire de milliers de citoyens qui voient en cette œuvre incomparable de Frank Lloyd Wright un symbole de la nation. Fallingwater est ainsi devenue la figure emblématique d'une américanité ancrée au génie du lieu. Le monument fait d'ailleurs l'apologie d'un architecte qui se décrit lui-même comme un « self-made man ».

À Poissy, tout se passe comme si la pensée de Le Corbusier habitait les lieux : «L'art dans un pays de haute culture, trouve son moyen d'expression dans l'œuvre véritable, concentrée et débarrassée de toutes fins utilitaires [...] » (Le Corbusier, 1995 : 112). Dans sa recherche d'équivalents formels de l'absolu, Le Corbusier a poursuivi une quête solitaire de la vérité. La villa Savoye, rapidement délaissée par ses occupants, a été prise en charge par une élite intellectuelle parisienne; ces « connaisseurs » se sont approprié le monument qui, à leurs yeux, incarne le mieux «l'émotion type » recherchée par l'architecte. De ce point de vue, l'icône, même assagie, doit rester intacte et surtout ne pas être spoliée par l'afflux de visiteurs ignares. La visite de la villa Savoye suppose en effet une connaissance préalable de la pensée - donc des écrits de Le Corbusier. La Villa aux heures claires est un «monument littéraire » qui s'adresse à la raison avant de toucher les sens. C'est aussi un monument aux prétentions universelles, tout aussi réfractaire aux constructions identitaires nationales qu'étranger à son milieu physique particulier.

Deux monuments fort différents donc, à la fois par leur genèse mais aussi par la lecture que le public peut en faire aujourd'hui, via des mises en tourisme culturellement très connotées.

«Seule la ville moderne offre à l'esprit le terrain où il peut prendre conscience de lui-même.»

Albert Camus

Gabriel Rioux est diplômé en urbanisme de l'UQAM où il poursuit des études supérieures à l'École des sciences de la gestion et au Département d'études urbaines et touristiques. Il est stagiaire associé à la Chaire de recherche du Canada en patrimoine urbain et c'est dans le cadre d'une mission sur la mise en valeur du patrimoine moderne qu'il a préparé cet essai.

\section{Notes}

1 «Le cantilever est, de tous les principes de constructions, le plus romanesque et le plus libre [...] ». Wright $(1998$ : 194) utilise la métaphore du plateau, maintenu en équili- 
bre par un serveur, pour illustrer le principe structurel du cantilever.

2 Comme en fait figure la couverture du Time en janvier 1938. On y voit Wright et, en arrière-plan, la perspective de la maison sur la Cascade.

3 « Il ne faut pas que le romantique dégénère en sentimental » (Wright, 1943).

4 Cet édifice représente pour Wright la forme la plus achevée de sa conception d'un espace sacré de travail. En complément, Fallingwater se veut l'espace idéal de l'habitat intégré à la nature.

5 Lors d'une discussion à la communauté de Taliesen, dans Pfeiffer, p.123.

6 Grammaire de Le Corbusier, comprenant ses célèbres cinq points : pilotis, le toit-jardin, le plan libre, la façade libre et les fenêtres en bandeau.

7 À ce sujet, consulter l'ouvrage de Peter Blake (1960).

8 Time, mars 1959

9 Il s'agit de la deuxième construction contemporaine remarquable à être classée en France au XXe siècle, après le théâtre des Champs-Élysées d'Auguste Perret.

10 Témoignage du cahier de Jean-Yves Le Guyader. L'album photo, toujours conservé à la médiathèque de l'Architecture et du patrimoine, témoigne avec éloquence de la dégradation avancée de l'édifice. Le lieu est méconnaissable jusqu'aux travaux de restauration dirigés par Yvan Gury en 1977.

11 Dans les faits, la villa a été reconstituée presque intégralement. Malgré des travaux attentifs, certains détails, comme la polychromie, suscitent toujours des controverses.

12 Appellation donnée, avec raison, par M. Savoye.

\section{Bibliographie}

Benton, Tim (1987), Les villas de Le Corbusier et Pierre Jeanneret, 1920-1930, New Haven, Yale University Press, 224 p.

Bergeron, Claude (1995), « L'architecture comme reflet de société. Un discours scientifique ou une mystification des historiens ? " dans Noppen, Luc, [dir.], Architecture, forme urbaine et identité collective, Sillery, Septentrion, $267 \mathrm{p}$.

Blake, Peter (1960), The Master Builders: Le Corbusier, Mies van der Rohe, Frank Lloyd Wright, New York, Norton, 1976, 430 p.

Boesiger, Willy, et Oscar G. Storonov (1937), Le Corbusier, Euvres complètes, Éditions d'Architecture, 1948, $214 \mathrm{p}$.
Caillot, Céline, et Ludovic Colas (1994), Le Pavillon Suisse - Cité internationale universitaire de Paris, Paris, École d'architecture de Paris, $18 \mathrm{p}$.

De Mignolla, France, et Cyril Delettre (2000), Autour de Poissy: Au pays des Yvelines, SPSA, $101 \mathrm{p}$.

Doremus, Thomas (1985), Frank Lloyd Wright and Le Corbusier: The Great Dialogue, New York, Van Nostrand Reinhold, 192 p.

Etlin, Richard A. (1994), Frank Lloyd Wright and Le Corbusier: The Romantic Legacy, New York, St-Martin's Press, 222 p.

Fishman, Robert (1977), L'utopie urbaine au $X X^{e}$ siècle, Bruxelles, Pierre Mardaga, 223 p.

Frampton, Kenneth (1985), Histoire critique de l'architecture moderne, Paris, Philippe Sers, $319 \mathrm{p}$.

Frampton, Kenneth (1997), Le Corbusier, Paris, Hazan, 198 p.

Gans, Deborah (1987), The Le Corbusier Guide, New York, Princetown Architectural Press, $192 \mathrm{p}$.

Hervé, Martin (1996), Guide de l'architecture moderne, Paris, Alternatives, 318 p.

Hoffmann, Donald (1978), Frank Lloyd Wright's Fallingwater: The House and History, New York, Dover, 98 p.

Journel, Guillemette Morel (1987), Le Corbusier, Paris, Actualité des arts plastiques no $72,82 \mathrm{p}$.

Journel, Guillemette Morel (1998), Collection indicateur du Patrimoine, La villa Savoye, Paris, Ministère de la Culture et de la Communication, direction du Patrimoine, $48 \mathrm{p}$.

Kaufmann, Edgar (1986), Fallingwater, a Frank Lloyd Wright Country House, New York, Abbeville Press, 190 p.

Kaufmann, Edgar (1989), 9 Commentaries on Frank Lloyd Wright, New York, The Architectural History Foundation, Mass., Cambridge, MIT Press, $158 \mathrm{p}$.

Le Corbusier, et P. Jeanneret (1927-1937), L'Esprit nouveau, 7 portfolios.

Le Corbusier (1923), Une petite maison, Zurich, Éd. d'architecture, $80 \mathrm{p}$.

Le Corbusier (1923), Vers une architecture, Paris, Champs-Flammarion, 1995, 253 p.

Levine, Neil (2000), dans Fallingwater and Pittsburgh, Narciso G. Menocal, 113 p.

Maddex, Diane (1985), Master Builders, Washington, National Trust for Historic Preservation, 203 p.

Massu, Claude (1990), Frank Lloyd Wright, Paris, Actualité des arts plastiques no 81, 81 p.
Noppen, Luc, et Lucie K. Morisset (1995), « De la production des monuments - Paradigmes et processus de la reconnaissance », dans Laurier Turgeon et al. [dir.], Les espaces de l'identité, Sainte-Foy, Presses de l'Université Laval, p. 2352.

Pfeiffer, Bruce Brooks (1994), Frank Lloyd Wright, Köln, Taschen, 175 p.

Pfeiffer, Bruce Brooks, et Gerald Nordland (1988), Frank Lloyd Wright in the Realm of Ideas, Southern Illinois University Press, 191 p.

Sanderson, Arlene (1991), Wright Sites, Illinois, Frank Lloyd Wright Building Conservancy, $56 \mathrm{p}$.

Sbriglio, Jacques (1999), Le Corbusier : La Villa Savoye, the Villa Savoye, Paris, Fondation Le Corbusier - Basel ; Boston ; Berlin, Birkhäuser, 187 p.

Stillman, Seymour (1948), Comparing Wright and Le Corbusier, p. 171-172, 175-178, 225234.

Storrer, William Allin (1990), The Architecture of Frank Lloyd Wright, Mass., Cambridge, MIT Press, n.p.

Tafuri, Manfredo et Francesco Dal Co (1976), Architecture contemporaine, Gallimard / Electra, 1991, 428 p.

Tietz, Jürgen (1999), Histoire de l'architecture du XX $X^{e}$ siècle, Cologne, Könemann, 120 p.

Time Magazine (1938), Usonian Architect, January 17, XXXI (3), La Une et p. 29-32.

Time Magazine (1959), Native genius, April 20, p. 56-57.

Time Magazine (1959), Stompin' on the Savoye, March 23, p. 66.

Toulier, Bernard (dir.) (1997), Collection indicateurs du Patrimoine, Mille monuments du XX $\mathrm{X}^{e}$ siècle en France, Paris, Ministère de la Culture et de la Communication, Direction du Patrimoine, $416 \mathrm{p}$.

Turner, Paul V. (1987), La formation de Le Corbusier : idéalisme et mouvement moderne, Paris, Macula, 259 p.

Veret, Jean-Louis (1990), « Passé, présent, futurs de la Villa Savoye » dans La Conservation de l'cuvre construite de Le Corbusier, Paris, Fondation Le Corbusier, p. 113-117.

Western Pennsylvania Conservancy (2000), Fallingwater, 16 p.

Wines, James (2000), L'architecture verte, Köln, Taschen, 240 p.

Wright, Frank Lloyd (1932), The Disappearing City, New York, W.F. Payson, 90 p.

Wright, Frank Lloyd (1943), Autobiographie, Paris, Les éditions de la Passion, 1998, 397 p.

Été 2002 Tégros 30

